\title{
Sensitive Determination of Nitroprusside in Blood by High Performance Liquid Chromatography
}

\author{
Toshinori Watanabe, Yuriko Nagamine, Toshihiko ToIda, Ichiro Koshishi and Toshio ImanarI
}

Faculty of Pharmaceutical Sciences, Chiba University, Yayoi-cho, Chiba 260

\begin{abstract}
A highly sensitive method for the determination of nitroprusside was developed by high performance liquid chromatography using an anion exchange column. In this method, nitroprusside was degraded to produce cyanide by heating in the presence of dithiothreitol, and the released cyanide was detected fluorometrically. A calibration curve for nitroprusside was linear in the range of 0.4 to 400 pmol. The usefulness and practicability of the present method were verified by application to the determination of nitroprusside in rat blood, which was infused to control the blood pressure.
\end{abstract}

Keywords High performance liquid chromatography, nitroprusside, cyanide, thiocyanate, biological materials

Sodium nitroprusside is widely used to keep hypotension during surgical operations ${ }^{1-2}$ and to reduce blood pressure immediately in the case of a hypertension emergency. ${ }^{3-4}$ The effectiveness of sodium nitroprusside is dependent on the following characteristic actions: blood pressure decreases just after sodium nitroprusside is administrated by i.v. infusion, and returns to the original state within a few minutes on stopping the infusion. ${ }^{5}$

However, nitroprusside is unstable in the presence of several biological components and liberate cyanide ${ }^{6-7}$, which may cause a severe toxic reaction. ${ }^{8}$ Monitoring the blood nitroprusside level is desirable for the safe therapy, but no sensitive and selective method for the determination of nitroprusside in blood has been reported.

Nitroprusside consists of five molar cyanides, one molar nitrosyl group and one molar ferrous ion. And it is immediately degraded into each component by heating in the presence of a large amount of thiols. ${ }^{9}$ If five molar cyanides liberated from one molar nitroprusside are determined, it is possible to determine sensitively the nitroprusside concentration in biological materials. Previously, we established a postcolumn HPLC method for the simultaneous determination of cyanide and thiocyanate using König reaction. ${ }^{10}$ In this paper, a high-sensitive and selective determination method of nitroprusside was established by incorporating its quantitative degradation procedure into the HPLC method which was used to determine cyanide in biological materials.

\section{Experimental}

\section{Reagents and materials}

All chemicals were of reagent grade. Reagent solutions were prepared by dissolving in deionized and distilled water. TSK gel QAE-2SW and TSK gel SAX were obtained from Toyo Soda Co. Ltd. (Tokyo). Asahipak BEST-502Q was obtained from Asahi Chemical Industry Co., Ltd. (Tokyo). The standard solution of nitroprusside $(10 \mathrm{mM})$ was prepared by dissolving sodium nitroprusside (Kanto Chemicals, Tokyo) in distilled and deionized water. A standard solution of cyanide $(0.1 \mathrm{M})$ was prepared from potassium cyanide (Wako Pure Chemicals, Osaka) by dissolving in $10 \mathrm{mM}$ sodium hydroxide solution, and was standardized by titration with silver nitrate according to the LiebigDénigès method.

\section{Chromatographic conditions}

The high performance liquid chromatograph used consists of four reciprocating pumps (PSU-2.5, Seishin Seiyaku Co., Ltd., Tokyo), a syringe-loading sample injector (Model 7125, Rheodyne Inc.), a variablewavelength fluorometer (RF-530, Shimadzu Co., Kyoto), a recorder (SS-250 F, Sekonic, Tokyo) and a water bath (Thermominder Jr-80, Taiyo Sciences Industrial Co., Tokyo). The established chromatographic conditions are shown in Fig. 1.

\section{Infusion of nitroprusside to rats}

Polyethylene tubes (i.d. $0.5 \mathrm{~mm}$, o.d. $0.8 \mathrm{~mm}$ ) were inserted to the femoral-vein and artery of Wister male rats $(200-300 \mathrm{~g})$, and an isotonic solution of nitroprus- 


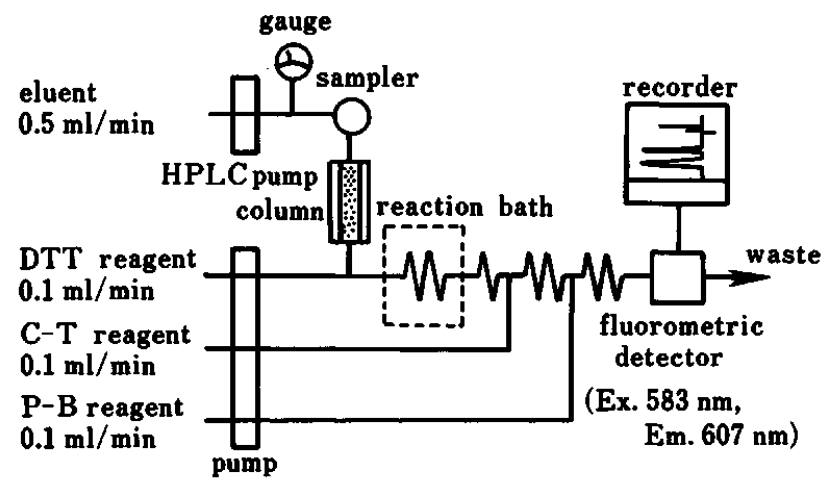

Fig. 1 Schematic diagram of high performance liquid chromatograph for determination of nitroprusside. Column, Asahipak BEST $502 \mathrm{Q}(4 \mathrm{~mm}$ i.d. $\times 150 \mathrm{~mm})$; eluent, $0.1 \mathrm{M}$ acetate buffer ( $\mathrm{pH}$ 6.0) containing $0.3 \mathrm{M} \mathrm{NaClO}$; DTT reagent, $5 \mathrm{mM}$ dithiothreitol in $0.05 \mathrm{M}$ Tris-HCl buffer ( $\mathrm{pH}$ 9.0) containing $5 \mathrm{mM}$ EDTA; C-T reagent, $0.5 \%$ Chloramine $T$ aqueous solution; $P-B$ reagent, mixture of $1.5 \mathrm{~g}$ barbituric acid, $15 \mathrm{ml}$ pyridine, $3 \mathrm{ml}$ concentrated hydrochloric acid and $82 \mathrm{ml}$ water; reaction bath, $90^{\circ} \mathrm{C}$; heating coil, $0.5 \mathrm{~mm}$ i.d. $\times 4.5 \mathrm{~m}$; sample volume, $20 \mu \mathrm{l}$.

side was infused into vein through a polyethylene tube. Artery blood was collected at each time from the femoral-artery through a polyethylene tube.

\section{Determination of nitroprusside in blood}

Sample preparation was carried out in a container shielded from a light on an ice bath just after the sample was collected. A $100 \mu l$ portion of whole blood or plasma was mixed with $100 \mu \mathrm{l}$ of $3.0 \mathrm{M}$ perchloric acid. After standing for $5 \mathrm{~min}$ in the ice-water bath, it was centrifuged at $8000 \mathrm{~g}$ for $5 \mathrm{~min}$. And then, $150 \mu \mathrm{l}$ of the supernatant was added to $150 \mu \mathrm{l}$ of $1.0 \mathrm{M}$ dipotassium hydrogenphosphate and the system was mixed vigorously. Excess perchloric acid was precipitated as potassium perchlorate by centrifugation at $8000 \mathrm{~g}$ for $5 \mathrm{~min}$. A $20 \mu \mathrm{l}$ portion of the supernatant was submitted to HPLC.

\section{Results and Discussion}

\section{Detection of nitroprusside}

The degradation of nitroprusside by heating in the presence of cysteine, glutathione or dithiothreitol (DTT) was investigated. Complete and brief degradation of nitroprusside was achieved by DTT in $50 \mathrm{mM}$ Tris- $\mathrm{HCl}$ buffer ( $\mathrm{pH} \mathrm{9.0)}$ containing $5 \mathrm{mM}$ ethylenediaminetetraacetic acid disodium salt (EDTA). The principle ${ }^{10-11}$ for the detection of nitroprusside is shown in Fig. 2. In this experiment, we examined the influence of DTT concentration and heating coil length on the degradation of nitroprusside using flow injection analysis. As shown in Fig. 3, nitroprusside was degraded in $50 \mathrm{mM}$ Tris- $\mathrm{HCl}$ buffer (pH 9.0) containing $5 \mathrm{mM}$ DTT and $5 \mathrm{mM}$ EDTA by passing through

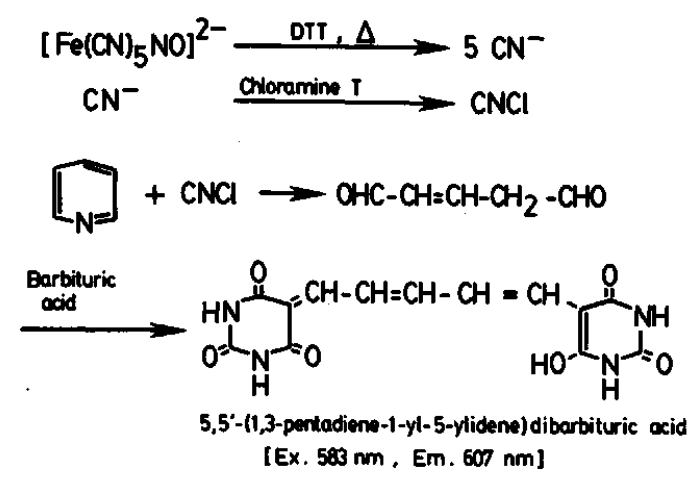

Fig. 2 Principle for the detection of nitroprusside.

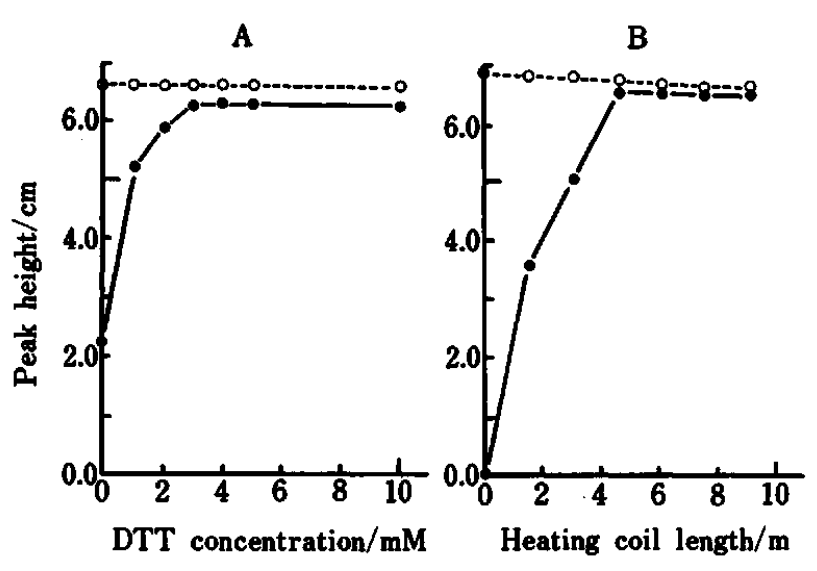

Fig. 3 Effects of DTT concentration and heating coil length on the degradation of nitroprusside. Eluent, $0.1 \mathrm{M}$ acetate buffer (pH 6.0); DTT reagent, A: DTT in $0.05 \mathrm{M}$ Tris-HCl buffer (pH 9.0) containing $5 \mathrm{mM}$ EDTA, B: $5 \mathrm{mM}$ DTT in $0.05 \mathrm{M}$ Tris-HCl buffer ( $\mathrm{pH}$ 9.0) containing $5 \mathrm{mM}$ EDTA; heating coil length, A: $4.5 \mathrm{~m}$; degradation temperature, $90^{\circ} \mathrm{C}$; detection, Ex. $583 \mathrm{~nm}$, Em. $607 \mathrm{~nm}$; sample, O: 2 $\mu \mathrm{M} N \mathrm{~N}, \mathrm{O}: 10 \mu \mathrm{M}$ cyanide; sample volume, $20 \mu \mathrm{l}$.

the degradation tube, $0.5 \mathrm{~mm}$ i.d. $\times 4.5 \mathrm{~m}$, at $90^{\circ} \mathrm{C}$. Furthermore, the five molar cyanides quantitatively liberated from one molar nitroprusside were measured.

\section{Separation of nitroprusside by HPLC}

Separation of nitroprusside from cyanide and thiocyanate, which are detectable by our method, was investigated by HPLC using anion exchange columns: Asahipak BEST-502Q (4 mm i.d. $\times 150 \mathrm{~mm})$, TSK gel QAE-2SW (4 mm i.d. $\times 150 \mathrm{~mm})$ and TSK gel SAX $(3 \mathrm{~mm}$ i.d. $\times 100 \mathrm{~mm})$. Typical separation of nitroprusside was achieved by using Asahipak BEST-502Q as the packing materials and sodium acetate buffer $\mathrm{pH}$ 6.0) containing sodium perchlorate as an eluent without interferences of cyanide or thiocyanate. The effects of sodium perchlorate concentration on the retention times of cyanide, thiocyanate and nitroprusside are shown in Fig. 4. A typical chromatogram is shown in Fig. 5. 


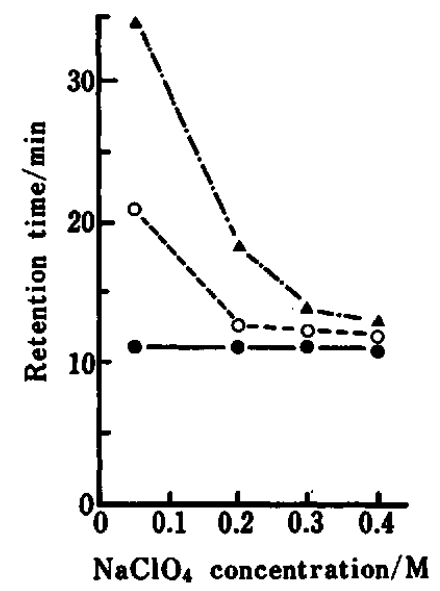

Fig. 4 Retention times of nitroprusside, cyanide and thiocyanate in various $\mathrm{NaClO}_{4}$ concentrations. cyanide; $O$, thiocyanate; $\Delta, N P$. Chromatographic conditions are as in Fig. 1.

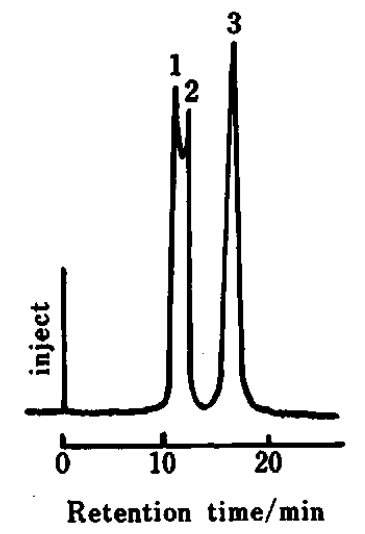

Fig. 5 Separation of cyanide, thiocyanate and nitroprusside by HPLC. 1, cyanide; 2 , thiocyanate; 3 , NP. Chromatographic conditions are as in Fig. 1.

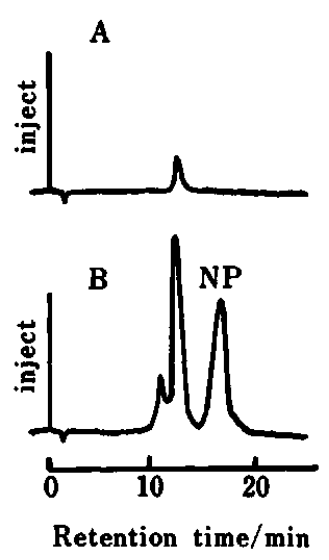

Fig. 6 Chromatograms of normal rat blood (A) and blood taken from rat infused nitroprusside (B). Sodium nitroprusside $(100 \mu \mathrm{g} / \mathrm{kg} / \mathrm{min})$ was infused intravenously to a male rat $(230 \mathrm{~g})$ for $10 \mathrm{~min}$, after which its arterial blood was taken. Chromatographic conditions are as in Fig. 1.
Table 1 Recovery tests of nitroprusside from rat blood and plasma

\begin{tabular}{cccc}
\hline & Added $/ \mu \mathrm{M}$ & Found $/ \mu \mathrm{M}$ & Recovery, \% \\
\hline Blood & 0.00 & N.D. & - \\
& 0.50 & 0.50 & 100 \\
& 1.00 & 0.97 & 97 \\
Plasma & 2.00 & 1.87 & 94 \\
& 0.00 & N.D. & - \\
& 1.00 & 0.99 & 99 \\
& 2.00 & 1.97 & 99 \\
\hline
\end{tabular}

N.D., not detected.

To make sure that the peak of nitroprusside corresponds to the composition $\left[\mathrm{Fe}(\mathrm{CN})_{5} \mathrm{NO}^{2-}\right.$, the nitrosyl group concentration was measured as a nitrous ion produced in alkaline solution by diazo-coupling reaction ${ }^{12}$ and the anion was completely recovered in the nitroprusside fraction.

The calibration curve for nitroprusside was linear in the range of 0.4 to $400 \mathrm{pmol}$; the detection limit was $0.2 \mathrm{pmol}$ (sample size, $20 \mu \mathrm{l}$ ). When $1.0 \mu \mathrm{M}$ nitroprusside was determined six times by the recommended procedure, the relative standard deviation was $1.6 \%$.

\section{Pretreatment of blood}

Instability of nitroprusside in the presence of hemoglobin and glutathione ${ }^{2}$ presents a serious difficulty for blood analysis. This is the reason why whole blood was mixed with high concentration of perchloric acid; nitroprusside was squeezed out from red blood cells and simultaneously proteins were precipitated. Details of pretreatment procedure for the determination of nitroprusside in blood were described in the experimental section. The chromatogram of blood taken from a rat, which was infused nitroprusside $(100 \mu \mathrm{g} / \mathrm{kg} / \mathrm{min})$ for $10 \mathrm{~min}$, is shown in Fig. 6. The analytical results of recovery tests from rat blood and plasma are shown in Table 1 . The recoveries of nitroprusside varied from 93.5 to $100.0 \%$.

\section{Application}

As mentioned above, blood pressure decreases as soon as nitroprusside is administrated by $i . v$. infusion, and returns to the original state within a few minutes on stopping the infusion. It is considered that these phenomena are dependent on the instability and the metabolic rate of nitroprusside in blood. Figure 7 shows that the nitroprusside level does not increase during the infusion period but is immediately steady, and rapidly decreases on stopping the infusion. The time-course of nitroprusside in arterial blood after the $i . v$. administration to rat is shown in Fig. 8. The halflife of nitroprusside level in rat blood was about $2.4 \mathrm{~min}$. These results correspond to the rapid return to original state on stopping the infusion. 


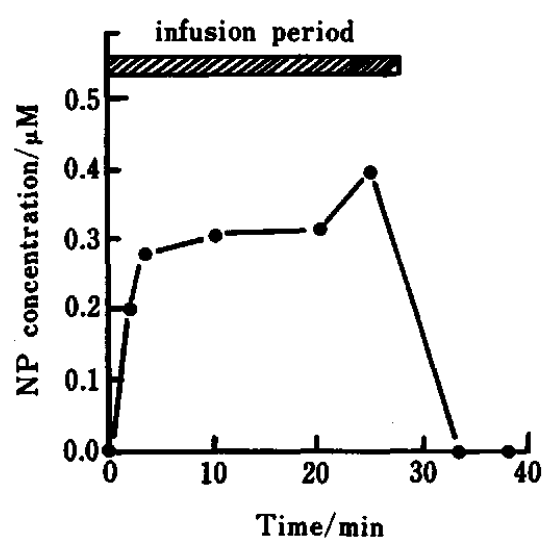

Fig. 7 Nitroprusside concentration in rat blood during and after infusion of NP. Sodium nitroprusside $(46.6 \mu \mathrm{g} / \mathrm{kg} /$ min) was infused intravenously to a male Wistar rat (235 g), after which its arterial blood was taken.

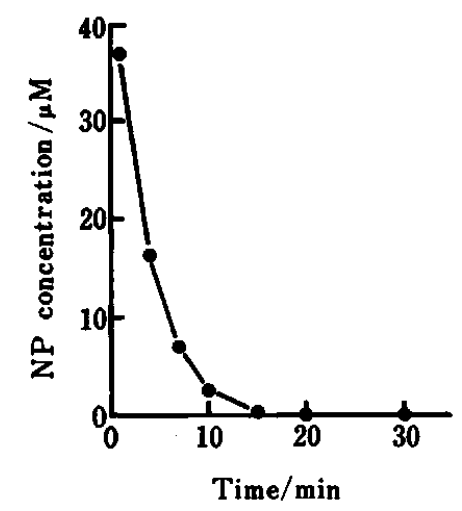

Fig. 8 Nitroprusside concentration in rat blood after administration of NP. Sodium nitroprusside $(4.0 \mathrm{mg} / \mathrm{kg}$ ) was administrated intravenously to a male Wistar rat $(300 \mathrm{~g})$, after which its arterial blood was taken.
Nitroprusside produces toxic cyanide during therapeutic usage and the metabolites must be monitored. However, the metabolic pathway of nitroprusside is not yet made clear. In this work, we have solved one of the most difficult problems in methodology for the investigation of nitroprusside metabolism.

\section{References}

1. P. Siegel, P. P. Moraca and J. R. Green, Br. J. Anaesth. 43, 790 (1971).

2. J. H. Tinker and J. D. Michenfelder, Anaesthesiology, 45, 340 (1976).

3. O. H. Page, A. C. Corcoran, H. P. Dustan and T. Koppanyl, Circulation, 11, 188 (1955).

4. V. Schlz and G. Loeschcke, J. Hypertension, 3, 485 (1985).

5. H. Schiffman and P. Fuchs, Acta Anaesth. Scand. (Suppl.), 23, 704 (1966).

6. C. J. Vesey, P. V. Cole and P. J. Simpson, Br. J. Anaesth., 48, 651 (1976).

7. Y. H. Kim, M. Foo and R. D. Terry, Arch Pathol. Lab. Med., 106, 392 (1982).

8. P. J. Bower and J. N. Petrson, N. Engl. J. Med., 293, 865 (1975).

9. F. L. Rodkey and H. A. Collison, Clin. Chem., 23, 1969 (1977).

10. T. Toida, T. Togawa, S. Tanabe and T. Imanari, $J$. Chromatogr., 308, 133 (1984).

11. T. Toida, Teikyo Med. J., 6, 163 (1983).

12. T. Toida, K. Kobayashi and T. Imanari, Bunseki Kagaku, 32, 130 (1983).

(Received October 3, 1987)

(Accepted January 9, 1988) 